Revue d'histoire de l'Amérique française

BRS REVUE D.HISTOIRE DE L'AMÉRIQUE FRANÇAISE

\title{
La mission de l'abbé Thomas Maguire à Rome, en 1833-1834
}

\section{Antonio Dansereau}

Volume 3, numéro 1, juin 1949

URI : https://id.erudit.org/iderudit/801523ar

DOI : https://doi.org/10.7202/801523ar

Aller au sommaire du numéro

Éditeur(s)

Institut d'histoire de l'Amérique française

ISSN

0035-2357 (imprimé)

1492-1383 (numérique)

Découvrir la revue

Citer cet article

Dansereau, A. (1949). La mission de l'abbé Thomas Maguire à Rome, en 1833-1834. Revue d'histoire de l'Amérique française, 3(1), 9-29.

https://doi.org/10.7202/801523ar d'utilisation que vous pouvez consulter en ligne.

https://apropos.erudit.org/fr/usagers/politique-dutilisation/ 


\section{LA MISSION DE L'ABBE THOMAS MAGUIRE A ROME, en 1833-1834}

En juin 1833, Mgr Signay, évêque de Québec, reçut de Rome une communication qui causa dans les milieux ecclésiastiques de la capitale une grande surprise. On lui apprenait le refus de la candidature de Mgr Turgeon au poste de coadjuteur de Québec, et on suggérait la nomination à sa place de M. St-Germain, curé de St-Laurent, près de Molntréal. Cette décision du Saint-Siège mettait l'évêque de Québec dans une position très délicate vis-à-vis du gouvernement anglais et des fidèles de son diocèse. Dans son premier mandement, en février 1833, Mgr Signay avait annoncé officiellement la nomination de Mgr Turgeon et la confirmation de ce choix par Lord Aylmer, gouverneur du Canada. Le nouvel élu avait aussitôt prêté le serment d'usage et commencé son administration. Ainsi l'application de la décision de Rome risquait de causer un véritable scandale. De plus la proposition de nommer M. St-Germain à la place de Mgr Turgeon n'avait rien de très rassurant. Depuis près de 12 ans, ce personnage avait créé aux évêques des ennuis très sérieux en soutenant la cause du Séminaire de Saint-Sulpice contre Mgr Lartigue, auxiliaire de l'évêque de Québec pour le district de Montréal. On s'explique alors facilement l'inquiétude de Mgr Signay en apprenant la décision de la Congrégation de la Propagande.

Mis au courant de l'affaire, Mgr Lartigue pressa l'évêque de Québec d'envoyer à Rome un délégué spécial, et proposa pour cette mission l'abbé Thomas Maguire, vicaire général, aumônier des Ursulines à Québec. Après plusieurs instances ${ }^{1}, \mathrm{Mgr}$ Signay accepta cette suggestion et, à la fin de septembre, M. Maguire partait pour Rome.

Le choix de ce délégué n'avait pas été fait au hasard. M. Maguire se recommandait par son expérience, par son caractère, par une con-

1. Correspondance de Mgr Lartigue de 1833 a 1836, Rapport de l'archiviste de la province de Québec (1943-1944): 222-240 (passim). 
naissance peu ordinaire des affaires canadiennes. En 1829-1830, Mgr Panet l'avait déjà chargé d'une mission à Londres et à Rome en vue d'obtenir l'érection du diocèse de Montréal et la révocation de l'autorisation d'aliéner ses seigneuries accordée à Saint-Sulpice ${ }^{2}$. Au cours de ses deux missions il se montra dévoué, courageux, audacieux même. Enfin ses mémoires et ses pétitions dénotent chez lui une connaissance très étendue et très solide des questions religieuses au Canada.

Il fallait tout cela pour aller défendre à Rome, en 1833, la cause des évêques de Québec, car leur agent allait se trouver en présence d'un adversaire assez redoutable en la personne de M. Jean-Baptiste Thavenet, P.S.S. Ce sulpicien français était bien connu au Canada pour y avoir résidé de 1794 à 1815. Après son retour en France, il avait été chargé a plusieurs reprises de la défense des intérêts de ses confrères canadiens à Londres et à Paris. A l'automne de 1830 , on l'envoya à Rome pour soutenir le point de vue du Sém'inaire de Montréal dans l'affaire de la nomination du curé d'office de la paroisse de Notre-Dame. Sa mission terminée, il y était resté sous le prétexte de composer un dictionnaire et une Histoire Sainte en algonquin pour le compte de la Propagande. En fait, s’il faut en croire M. Maguire, sans mandat officiel et malgré la défense expresse de son supérieur, il continuait de fournir au Saint-Siège des informations plus ou moins exactes sur les affaires religieuses du Canada ${ }^{3}$. Grâce à ses intrigues et à ses largesses, il avait libre accès auprès des secrétaires des Congrégations romaines et pouvait facilement faire prévaloir son point de vue. Toujours d'après $M$. Maguire, il aurait même réussi à prendre connaissance de la correspondance secrète des évêques du Canada avec Rome. C'est lui qui avait fait échouer la candidature de $\mathrm{Mgr}$ Turgeon et avait proposé à sa place M. St-Germain.

M. Maguire allait donc se trouver dans une situation assez difficile pour défendre Mgr Turgeon et les autres causes de l'évêque de Québec contre le Séminaire de Montréal.

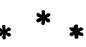

2. Abbé Ivanhoë Caron, "Les évêques de Québec, leurs procureurs et leurs vicàires généraux, à Rome, à Paris et à Londres (1734-1834)". Memoires de la Société Royale, section I, (1935): 153-178.

3. Maguire à Mgr Signay, 17 février 1834. Archives de l'Archevêché de Montréal, dossier Maguire. Cette source sera désignée dans la suite par les lettres AAM. 
Dès son arrivée à Rome, le 5 décembre 1833 , il se mit résolument a la tâche. Le premier objet de sa mission était l'obtention des bulles de Mgr Turgeon. La lutte fut longue et difficile. Il dut présenter successivement trois requêtes. Avant de produire la première, il fit une enquête pour connaître la nature et la source des objections contre la nomînation de $\mathrm{Mgr}$ Turgeon. Ses premières visites furent pour $\mathrm{M}$. Thavenet. "Je n'ai eu rien de plus pressé à mon arrivée ici que de le voir pour prendre hauteur ", écrivit-il dans sa première lettre à $\mathrm{Mgr}$ Signay. Malgré un accueil bienveillant, il ne réussit pas a obtenir d'autres réponses que celle-ci: " La question de M. Turgeon ne pouvait être traitée d'abord; il fallait au préalable que toutes les autres difficultés fussent terminées à l'amiable, et d'une manière satisfaisante pour les intéressés "). Son enquête se poursuivit auprès des membres de la Congrégation de la Propagande: les cardinaux Pédicini, Weld, Fesch, le secrétaire Mgr Mai, le sous-secrétaire Mgr Palma. Sur la nature des objections contre Mgr Turgeon la réponse fut invariablement la même: "Tous au premier abord et sans détour m'ont informé que cette élection ne pouvait être confirmée, parce que ce monsieur était opposé aux sulpiciens de Montréal et était ennemi de leur congrégation. A cette question: par quelle voie la Cour de Rome reçoit-elle ses informations? on a répondu: par l'entremise de M. l'abbé Thavenet, son agent " . " Non satisfait de ces réponses laconiques, visiblement inspirées de M. Thavenet, il écrivit alors une lettre officielle à la Propagande pour obtenir de plus amples informations. Il posait les questions suivantes: "Quelles sont les objections canoniques que l'on oppose à l'élection de M. l'abbé Turgeon? et quels sont les individus qui les font valoir? - Par quelle voie ces objections sont-elles connues du S. Siège? - Y a-t-il à Rome auprès du S. Siège quelque agent de S. Sulpice de Montréal? ou de quelque autre corporation ou individu du diocèse de Qúébec? et quel est cet agent $?^{5}$ ) - Trois jours après, M. Maguire recevait du secrétaire de la Propagande cette réponse vraiment surprenante: "Comme les difficultés entre l'archevêque de Québec et S. Sulpice devaient être terminées à l'amiable, il ne pouvait fournir aucun des renseignements demandés, excepté que Sa Sainteté avait fait notifier officiellement à $M$. Thavenet qu'elle le nommait

4. Maguire à Signay et à Lartigue, 8 décembre 1833, AAM.

5. Maguire a Mgr Mai, secrétaire de la Propagande, 9 décembre 1833, AAM. 
agent de S. Sulpice pour la présente occasion ") ${ }^{6}$. Il fut donc obligé de se contenter de ces maigres renseignements, et c'est dans ces conditions peu rassurantes qu'il présenta au S.-Siège la première pétition de Mgr Signay en faveur de Mgr Turgeon.

Voici en substance ce qu'elle contenait. Sensiblement affligé par les délais du Saint-Siège à accorder l'institution canonique à $\mathbf{M g r}$ Turgeon, inquiet d'un projet de substitution de M. St-Germain à Mgr Turgeon, appuyé et encouragé par le clergé et les laïcs de son diocèse, l'évêque de Québec s'est décidé à envoyer à Rome M. Thomas Maguire pour exposer très respectueusement les raisons pour lesquelles il semble nécessaire de donner à Mgr Turgeon l'institution canonique. Ces raisons sont les suivantes: 10 - Un plus long délai créerait un scandale, parce que Mgr Turgeon, reconnu officiellement par le gouvernement britannique, a prêté le serment d'usage et partage déjà avec l'évêque l'administration du diocèse; 20 - il est impossible de revenir sur l'élection de Mgr Turgeon avec un gouvernement protestant, qui s'arroge le droit d'intervenir dans la nomination des évêques; c'est l'opinion, non seulement de l'évêque de Québec, mais aussi de toutes les personnes bien au courant de la situation religieuse au Canada, en particulier des évêques de Montréal, de Kingston, de New-York; 30 - la candidature de Mgr Turgeon " a réuni les suffrages de tout le clergé et peuple canadiens sans qu'une seule voix se soit élevée contre sa nomination ". A la suite des informations recueillies à Rome, M. Maguire crut bon d'ajouter à ce dernier argument deux remarques concérnant l'attitude réciproque de $\mathrm{Mgr}$ Turgeon et de la Congrégation de Saint-Sulpice de Montréal. Cette dernière ne s'oppose pas à l'élection de Mgr Turgeon, puisque le supérieur provincial a désavoué formellement « toute agence et toute intervention, soit à Rome, soit au Canada, relativement à l'élection du Coadjuteur de Québec ". De son côté, Mgr Turgeon n'est pas hostile à la Congrégation de Saint-Sulpice. Il a toujours été opposé, il est vrai, à la cession des biens du Séminaire de Montréal au gouvernement canadien; mais il partage cette attitude " avec les évêques, le clergé et la masse entière du peuple; et de plus avec l'une des branches de la Législature, la Chambre d'Assemblée, autorité suprême dans le BasCanada ). ${ }^{7}$

6. Maguire à Signay et à Lartigue, 3 janvier 1834, AAM.

7. Pétition de M. Maguire à Grégoire XVI, 16 décembre 1833, AAM. 
Cette première requête manque presque complètement son but, parce que M. Maguire ignorait le vrai motif de l'opposition de SaintSulpice à la nomination de Mgr Turgeon. Il l'apprit de M. Thavenet lui-même, quelques jours après la communication de sa pétition au Saint-Siège. Voici comment il annonça le fait à Mgr Signay:

Votre Grandeur sera étonnée sans doute d'une communication que $M$. Thavenet $m$ 'a faite ces jours derniers et qui dévioile le mystère du refus des bulles de M. Turgeon. La voici. M. Turgeon, dans une conférence avec M. Quiblier, ${ }^{8}$ lui a déclaré que pour ramener la paix entre les évêques et Saint-Sulpice, il fallait de toute nécessité que ce dernier devint canadien. Mais le Pape et les Cardinaux à Rome, et les Sulpiciens à Paris, dø̈cident, suivant $M$. Thavenet, que l'œuvre de la fondation de St-Sulpice de Montréal ne peut en conscience (exc'epté dans les cas extrêmes) être acquittée que par des Français, et que cette Maison doit toujours être gouvernée que par une majorité de Français. Donc, M. Turgeon qui veut que cette maison soit canadienne est inéligible à l'épiscopat'.

Toute l'affaire se ramenait donc au problème de l'introduction de prêtres français au Séminaire de Montréal. Aussi M. Maguire, qui n'avaít pas prévu cette difficulté, appréhendait-il une décision défavorable. De fait, dans la Congrégation du 27 janvier, les Cardinaux recommandèrent au Saint-Père " de n'accorder ses bulles à $M$. Turgeon qu'après une promesse formelle de sa part, ou de la part de son agent à Róome, de ne s'opposer jamais à l'introduction de prêtres françaìs au Séminaire de Montréal. ") ${ }^{10}$

Cette décision produisit sur M. Maguire une très forte impression. De suite, il se rendit chez le secrétaire de la Propagande pour lui " témoigner dans les termes les plus forts son étonnement qu'une poignée d'étrangers soient ainsi soutenue dans une espèce de rébellion contre leurs supérieurs légitimes, au point d'entraver la succession à l'épiscopat et d'exposer l'Eglise du Bas-Canada à des malheurs irréparables " ${ }^{11}$. Mgr Mai lui répondit que, si Sain t-Sulpice était vraiment

8. M. Vincent Quiblier était supérieur du Séminaire de Montréal depuis 1831.

9. $M$ aguire à Signay et Lartigue, 3 janvier 1834, AAM. AMM.

10. Cardinal Pédicini, préfet de la Propagande, à M. Maguire, 1 février 1834,

11. Maguire à Signay et à Lartigue, 6 février 1834, AAM. 
coupable, on pourrait suggérer l'envoi des bulles sans exiger de $\mathrm{Mgr}$ Turgeon une promesse formelle, mais en lui recommandant simplement de ne pas mettre obstacle à l'entrée de sulpiciens français à Montréal. En même temps le secrétaire l'invitait à exposer par écrit les griefs de l'évêque de Québec contre Saint-Sulpice.

De retour chez lui, M. Maguire écrivit d'abord au Cardinal Pédicini pour lui dire qu'il ne pouvait en conscience faire la promesse demandée, parce qu'il avait l'intime persuasion que M. Turgeon luimême ne la ferait pas, et que d'ailleurs il n'avait reçu aucune instruction sur ce point. ${ }^{12}$

Trois jours après, il présentait a Mgr Mai un long réquisitoire contre le Séminaire de Montréal ${ }^{13}$. Après avoir exprimé sa douleur à la vue des dangers qui menacent l'Église du Canada, et son étonnement devant l'appui que reçoivent a Rome des prêtres étrangers au pays et rebelles aux évêques, il réfute quelques-unes des informations mensongères fournies à la Propagande par $M$. Thavenet. Ce dernier avait soutenu que " l'œuvre de la fondation de S.-Sulpice ne pouvait être acquittée que par des sulpiciens français 》. A cette affirmation, M. Maguire oppose l'avis de juristes éminents ${ }^{14}$ et de Saint-Sulpice lui-même ${ }^{15}$ qui avaient démontré clairement " l'entière séparation \& totale indépendance " du Séminaire de Montréal et de celui de Paris par la seule loi de conquête. D'ailleurs, de 1760 à 1794, Saint-Sulpice de Montréal s'était recruté uniquement au Canada et pour le plus grand bien du pays. - $M$. Thavenet avait accusé les évêques de Québec de vouloir " s'emparer des biens du Séminaire de Montréal, comme ils avaient envahi ceux du séminaire des Missions Etrangères de Québec ". M. Maguire repousse cette accusation avec indignation; " Jamais, dit-il, les évêques n'ont touché à un denier des biens du Séminaire de Québec ". Quant au Séminaire de Montréal, il serait impossible de le déposséder avec les lois qui régissent les propriétés

12. Maguire au cardinal Pédicini, 3 fév. 1834, AAM.

13. Maguire à Mgr Mai, 5 fév. 1834, AAM.

14. Avec sa pétition M. Maguire communiqua à la Propagande la brochure où se trouve exprimée l'opinion des juristes; elle s'intitule: Consultation de M. Dupin, avocat de la Cour Royale de Paris, pour le Séminaire de Montréal, en Canada (Paris imprimerie d'Everat, 1826).

15. L'opinion de Saint-Sulpice se trouve exprimée dans le Mémoire pour le Séminaire de Montréal, brochure anonyme, sans lieu et sans date, attribuée à $\mathbf{M}$. Roux; elle fut communiquée à la Propagande avec la Pétition. 
de main-morte au Canada, et surtout avec un gouvernement qui prétend avoir des droits sur ses biens. - $M$. Thavenet avait encore dit que « S. Sulpice jouit d'une grande considération dans le Canada et que les Evêques ne devraient pas abattre cet établissement". A cela M. Maguire répond en affirmant " de la manière la plus solemnelle " que jamais les évêques n'ont essayé de supprimer le Séminaire de Montréal. Pour ce qui est de la réputation de Saint-Sulpice, elle est " nulle, absolument nulle " à cause de l'attitude des sulpiciens frạnçais a l'égard des évêques, depuis 1821. D'ailleurs, avant cette date, sa réputation était surfaite; tout compte fait, au Canada, SaintSulpice ne dirige qu'une paroisse, une petite bourgade d'Indiens et un collège, qui, depuis quelques années, tombe rapidement dans l'opinion publique. ${ }^{16}$

Après ces observations, M. Maguire aborde le sujet principal de sa requête: les inconvénients de l'introduction de prêtres français au Séminaire de Montréal. Les évêques de Québec ne peuvent l'autoriser sans encourir l'indignation du gouvernement anglais. Ce dernier a déjà manifesté son hostilité à l'égard d'une institution qui ne peut se recruter que chez l'ennemi. Tout dernièrement encore, Lord Goderich écrivait à l'évêque de Québec: " Je concours de plus en plus avec mes prédécesseurs pour blâmer la continuation d'une institution qui au lieu de remplir par ses propres élèves (sujets anglais) les vacances qui ont lieu dans son corps, a été obligée de recruter ses forces parmi un clergé appartenant à un pays étranger qui est attaché au Bas-Canada par les liens du langage, de l'origine et de l'ancienne sujestion ". D'un autre côté, cette pratique menace le Séminaire de Montréal dans la possession de ses biens. Chaque fois que Saint-Sulpice veut faire venir de France de nouveaux sujets, le gouvernement anglais demande impérieusement la cession de ses biens. - Enfin, le privilège concédé à Saint-Sulpice de Paris d'envoyer des prêtres de son choix, "à l'insu et même contre le gré des premiers pasteurs "), est de nature à occasionner de graves abus. En fait, cette coutume a contribué à entretenir la discorde dans l'Église du Canada et spécialement dans le district

16. M. Maguire se garde bien de dire ici que la paroisse de Notre-Dame était de beaucơup la plus importante du Canada, comprenant la ville de Montréal et sa banlieue avec une population d'environ 40,000 âmes. Quant au Collège, il avait perdu de son prestige, surtout parce que les professeurs français manifestaient un peu trop ouvertement leur opposition aux principes démocratiques du parti patriote de Louis-Joseph Papineau. 
de Montréal. En 1821 spécialement, le Séminaire de Saint-Sulpice de cette ville, dirigé par des prêtres français, a fomenté « un schisme (et le m'ot est dit avec réflexion) ", en s'opposant à l'ordre établi par le Saint-Siège pour le gouvernement spirituel de cette région. Depuis, le " schisme " a continué: "Jamais S. Sulpice n'a eu recours pour le spirituel aux évêques. - Au vu \& au su de la population entière de Montréal, jamais de rapports avec l'Évêque du District, pour les devoirs même des bienséances et de la vie civile, quoique celui-ci ait constamment, surtout avant ses infirmités, fréquenté leur Maison.Jamais d'assistance de la part des Sulpiciens aux cérémonies épiscopales, même des ordinations de leurs élèves. etc, etc... ".

A la fin de son réquisitoire, $M$. Maguire annonce qu'il vient de recevoir du Canada une nouvelle importante: le Clergé canadien est en train de préparer une pétition en faveur de Mgr Turgeon. Malheureusement le document officiel ne pourra pas parvenir à Rome avant trois ou quatre mois; mais cette simple information devrait être quand même d'un poids considérable en faveur de l'évêque de Québec.

Obligé de présenter ce mémoire avant d'avoir reçu les instructions nécessaires, par ailleurs vivement impressionné par la décision de la Propagande, M. Maguire ne sut pas se garder complètement des exagérations et des préjugés répandus au Canada contre Saint-Sulpice. L'exagération est manifeste quand il affirme que la réputation de Saint-Sulpice est " nulle, absolument nulle "; ou bien encore lorsqu'il qualifie de schisme les difficultés entre le Séminaire de Montréal et Mgr Lartigue. Dans la dernière partie de son mémoire, presque chacune de ses affirmations demanderait une mise au point qu'il serait trop long de faire ici. Il suffira de dire que Mgr Lartigue, à qui il envoya une copie de sa lettre, se crut obligé de lui signaler les principales erreurs et de lui suggérer une rectification. ${ }^{17} \mathrm{M}$. Maguire lui répondit: " Je rectifierai autant que possible (car la vérité avant tout) les impressions causées par mes assertions exagérées, mais faites de bonne foi, relativement à S.-Sulpice; je puis cependant dire que mes jamais me sont venus presque tous de St-Jacques ${ }^{18}$

17. Lartigue à Maguire, 29 avril 1834, Rapport de l'Archiviste de la province de Quebec, (1943-1944): 262-263.

18. Maguire à Lartigue, 6 juillet 1834. - St-Jacques désigne ici l'ancien évêché de Montréal, résidence de Mgr Lartigue. 
Toutefois sa rétractation fut trop tardive pour exercer une influence sur la décision du Saint-Siège. Dès le 15 février, le secrétaire de la Propagande communiqua au Saint-Père, avec la recommandation des Cardinaux, tous les documents de M. Maguire. Grégoire XVI tenait à être informé personnellement de la question. Avant son élevation au Souverain Pontificat, il avait été, pendant de longues années, Préfet de la Sacrée Congrégation de la Propagande. Il connaissait à fond tous les problèmes de l'Église catholique au Canada et se réservait de leur donner une solution définitive. Dans le cas de Mgr Turgeon, il passa trois heures à examiner et discuter les papiers de M. Maguire. Il décida finalement d'accorder les bulles sans condition; mais, par égard pour les Cardinaux et pour Saint-Sulpice, elles seront accompagnées d'une lettre recommandant fortement au nouveau coadjuteur de ne pas s'opposer à l'introduction de Sulpiciens français a Montréal.

Dès le lendemain, M. Maguire fut mis au courant de la décision. En même temps on l'invitait à écrire de nouveau au Saint-Père. Il le fit cette fois sur un ton plus modéré et plus brièvement, se contentant d'exposer sous une autre forme les inconvénients du recrutement du Séminaire de Montréal en France. ${ }^{19} \mathrm{~L}$ 'effet fut d'atténuer davantage les exigences du Saint-Siège. Dans la lettre de recommandation accompagnant les bulles, il ne fut plus question de l'admission de prêtres français a Montrél, mais d'une simple exhortation à la paix avec Saint-Sulpice. ${ }^{20}$ C'était pour M. Maguire une victoire complète.

La deuxième cause que le délégué de l'évêque de Québec avait pour mission de défendre était celle des biens du Séminaire de Montréal. Depuis quelques années, cette question causait de sérieuses inquiétudes aux évêques du Canada. Par des moyens plus ou moins violents, le gouvernement anglais cherchait à s'emparer de ces biens pour en doter les institutions protestantes. Après bien des instances, la direction du Séminaire avait fini par accepter les propositions du gouverneur qui consistaient dans l'échange des seigneuries contre une rente annuelle. En 1827, à l'insu de l'évêque de Québec, M. Roux, supérieur provincial, se rendit en Europe, obtint de Rome l'autorisation néces-

19. Maguire à S.S. Grégoire XVI, 18 fév. 1834, AAM.

20. Maguire à Signay et Lartigue, 12 mars 1834, AAM. 
saire et entama les négociations avec Londres. Mis au courant de l'affaire, Mgr Panet délégua aussitôt à Rome M. Antoine Tabeau, curé de Boucherville, et $M$. Maguire dans le but d'obtenir le retrait de l'autorisation. Le Souverain Pontife reconnut le bien-fondé de leur réclamation et ordonna à M. Roux de suspendre les négociations ${ }^{21}$. Mais, en 1832, l'affaire reprit son cours à la suite d'une nouvelle démarche du gouvernement anglais qui, maintenant, demanda it l'échange des propriétés du Séminaire contre des biens immeubles.

Cette fois encore, M. Maguire fut chargé de faire échouer le projet. Au nom de l'évêque de Québec, il devait supplier le Souverain Pontife de vouloir bien " signifier dans le plus court délaj à la compagnie de S.-Sulpice de Montréal une défense formelle de vendre ou céder ses seigneuries, fiefs ou biens-fonds soit au Gouvernement Britannique ou à d'autres sans l'intervention et le consentement exprès du dit Seigneur Archevêque ou de ses successeurs, suivant les lois civiles et ecclésiastiques en force dans le Canada 1). 22

Dans la pétition qu'il présenta au S.-Siège, il s'appliqua surtout a démontrer que la transaction projetée pourrait bien être le point de départ d'une confiscation générale des biens de l'Église catholique au Canada. C'était l'avis de Mgr Plessis, en 1819, de la Chambre d'Assemblée du Bas-Canada, de Saint-Sulpice lui-même qui, dans un mémoire publié en 1819 et attribué à M. Roux, s'exprimait ainsi:

Aucun bien public ne paraîtra dans l'envahissement de biens totalement employés au bien public: on ne verra que le plan de substituer des Missionnaires, un Collège, des Ecoles Protestantes, à des Missionnaires, à un Collège, à des Écoles Catholiques. Après avoir vu successivement tomber Jésuites et Récollets, c.a.d. cinq 6́tablissements religieux; encore celui de St-Sulpice, rempli de sujets, tomber, le peuple ne verrait que la haine de ses établissements Catholiques, il les verrait successivement frappés; bientôt, le Séminaire de Québec, les hôpitaux, les maisons d'éducation, la dime des Curés etc... toute la Religion Catholique s'( écrouler ) $)^{23}$.

21. Abbé Ivanhoë Caron, "Les évêques de Québec, leurs procureurs et leurs vicaires généraux, à Rome, à Paris et à Londres (1734-1834)", M'émoires de la Société Royale, section I, (1935): 171.

22. Pétition de M. Maguire à Grégoire XVI, 26 fév. 1834, AAM.

23. Considérations sur les Biens du Séminaire de Montréal, p. 16. - Brochure anonyme, sans lieu et sans date, attribuée à M. Roux. Elle fut communiquée a la Propagande avec la Pétition. 
D'un autre côté, la conduite du gouvernement anglais depuis la conquête permettait de croire que la confiscation des biens du Séminaire de Montréal faisait partie d'un plan d'ensemble consistant a dépouiller graduellement les institutions catholiques au profit des institutions protestantes.

1o - Le Gouvernement Britannique a enlevé aux Recollets leurs établissements catholiques et les a affectés en partio au culte protestant. 20 - Il a diverti pendant les trente dernières années les biens des Jésuites, destinés comme ceux de S. Sulpice à l'instruction catholique des fidèles et à la conversion des aborigènes du pays; il en a bâti de superbes temples protestants, salarié des ministres protestants, 6tabli des écoles protestantes; et ce n'est qu'après 20 ans d'efforts perséverants que les Communes du Bas-Canada ont enfin obtenu la restitution de ces biens au pays, mais non à la religion quoique celle-ci y eût des droits sacrés. 3o - Il a enlevé sans formalités quelconques et de vive force des terrains d'une valeur très considérable à deux monastères de la ville de Québec, l'un de Religieuses Ursulines et l'autre d'Hospitalières. 4o - Il retient aux mêmes religieuses Ursulines plus de 8,000 arpents de terre d'une seigneurie, malgré leurs réclamations depuis près de 20 ans.

Enfia, dans ses dernières propositions a Saint-Sulpice, le gouvernement anglais s'exprimait assez clairement sur ses intentions:

Quant au surplus des revenus provenant des biens de $\mathbf{S}$. Sulpice, après qu'on aura pourvu aux articles del'arrangement, ce surplus sera appliqué à des objets qui approcheront autant que possible des charges de la fondation, c.a.d. à l'education genérale ou à l'instruction religieuse, et en conséquence vous mettrez ce surplus à la disposition de la Législature provinciale, précisément de la même manière que l'on a fait pour les biens des Jésuites. ${ }^{24}$

A propos de cette dernière demande, M. Maguire fait remarquer que la Législature du Bas-Canada est composée de trois branches dont deux a majorité protestante; elles ne manqueraient pas de doter d'abord les institutions protestantes " ou des écoles libérales; dont l'enseignement serait basé sur des principes hétérogènes qui prédominent de nos jours ").

24. Dépêche du ministre des Colonies à Lord Aylmer, AAM. 
Quant au dernier projet du gouvernement qui consistait a vouloir échanger toutes les propriétés du Séminaire contre des biens immeubles, M. Maguire pensait qu'il était irréalisable. Le gouvernement anglais ne possédait ni les immeubles offrant une compensation suffisante, ni la somme d'argent ( 2 millions de francs) nécessaire à leur acquisition. Il y avait bien les terres de la Couronne; mais, situées en des lieux sauvages et inaccessibles, elles ne pourraient rien donner, si ce n'est dans un avenir très reculé.

Deux lettres adressées au secrétaire de la Propagande vinrent compléter les informations contenues dans ce mémoire. La première annonçait que l'on vendit de déposer à la chambre des Communes du Bas-Canada un projet de loi ayant pour effet d'empêcher la confiscation des biens du Séminaire de Montréal; preuve de l'importance que l'on attache au Canada à leur conservation ${ }^{25}$. L'autre lettre avait pour but de rectifier une information inexacte de $M$. Thavenet sur les revenus de S.-Sulpice. Il avait prétendu que le Séminaire de Montréal pouvait céder ses seigneuries sans inconvénients, à condition qu'on lui laissât ses biens-fonds d'un revenu annuel de 50,000 francs. Or, dans le Rapport de 1819, on note que ces propriétés (2 fermes et 3 moulins) ( rapportent si peu de chose qu'il ne vaut pas la peine de les faire entrer en ligne de compte ". D'ailleurs cette partie des domaines du Séminaire était menacée comme tout le reste, le gouvernement anglais exigeant la cession de tous les biens sans distinction. Pour éviter un pareil malheur il suffirait "d'un ordre simple mais bien formel a S.-Sulpice de n'aliéner ses biens qu'àvec l'agrément de l'Archevêque ét suivant les lois du pays " ${ }^{26}$

A la Propagande, cette requête ne rencontra aucun obstacle. Dans la Congrégation du 21 juillet, les Cardinaux la recommandèrent au Souverain Pontife et ce dernier ratifia leur décision. Ils demandèrent seulement que l'on usât de beaucoup de ménagement à l'égard de Saint-Sulpice; " les Cardinaux Sulpiciens désirent que cette defense soit couchée dans le langage le plus poli... Le Saint-Père est de cet avis et moi j'ai demandé qu'elle fut signifiée par le canal des évéques $",{ }^{27}$ ce qui fut accordé.

25. Maguire a Mgr Mai, 1 avril 1834, AAM.

26. Mguire à Mgr Mai, 23 avril 1834, AAM.

27. Maguire a Signay et Lartigue, 28 juillet 1834, AAM. 
C'est aussi dans la Congrégation du 21 juillet que les Cardinaux rendirent leur jugement sur le mode d'élection des coadjuteurs de l'évêque de Québec. Ce problème délicat se posa en 1826, quand le Saint-Siège, pour des raisons d'ordre canonique, voulut modifier la coutume établie depuis la conquête. Cette coutume laissait le choix du candidat à l'évêque de Québec, qui, avec l'approbation du gouverneur canadien, demandait ensuite les provisions en Cour de Rome. Pour éviter tout difficulté avec le gouvernement anglais, le Saint-Siège avait dabord approuvé ce mode de nomination. Mais, en 1826, profitant de l'élection de Mgr Signay, il voulut en introduire un autre consistant dans la présentation de trois candidats parmi lesquels le Souverain P'ontife lui-même choisirait le coadjuteur. Etant donné l'attitude du gouvernement anglais sur la question de l'épiscopat au Canada, ce nouveau procédé présentait de sérieux inconvénients. Aussi en 1833, Mgr Sïgnay chargea-t-il son délégué d'exposer la situation au Saint-Siège et de proposer les modifications nécessaires.

Dans sa pétition, M. Maguire s'efforce de montrer le danger réel qui menace la succession épiscopale à Québec par suite des prétentions anglaises. Sa Majesté Britannique a constamment réclamé le droit de nommer les évêques, soit en vertu de la suprématie spirituelle attachée à sa couronne, soit en vertu du droit de conquête ou comme successeur des rois de France. Non seulement le gouvernement anglais a fait inscrire ce droit dans les constitutions, toujours en vigueur, de 1774 et de 1791, mais il le réaffirme dans les instructions secrètes données à chaque gouverneur depuis 1775 . Un des articles se lit comme suit: "

...Afin que notre juste SUPREMATIE dans les matières tant ECCLESIASTIQUES que civiles puisse avoir son plein et entier effet Nous voulons et c'est Notre bon plaisir:

1o Que tous appels à aucune jurisdiction étrangère (i.e. Rome) ou correspondance avec icelle de quelque nature qu'ils puissent être, soient absolument défendus, et ce sous les pénalités les plus sévères. 2o Que les pouvoirs épiscopaux ou vicariaux ne soient exerces dans notre dite Province par aucune personne... sans une LICENCE ET PERMISSION de vous sous le sceau de notre dite Province et seulement pour le temps de notre BON PLAISIR, et sous telles limitations et restrictions qui correspondent à l'esprit et aux clauses de l'acte du PARLEMENT de la 14e année de Notre règne 
(acte de 1774)... et qu'aucune personne ne puisse recevoir les ORDRES SACRÉS ni prendre CHARGE D'ÂMES, qu'après avoir obtenu de vous une permission ecrite...

Ces prétentions apparaissent encore dans la conduite du gouvernement anglais et de ses représentants, depuis 1760 . Par politique, il est vrai, ils ont do, la plupart du temps, adopter des procédés de conciliation. Mais, chaque fois que l'occasion se présenta, ils ne reculèrent pas devant des moyens plus ou moins violents pour réduire l'Eglise du Canada sous la suprématie de Sa Majesté Britannique. Par exemple: " 10 Le Siège de Québec resta vacant pendant six années consécutives après la conquête du Canada, au grand détriment de la religion. - 2o Le Gouvernement refusa 60 ans durant de reconnaitre, du moins ouvertement, comme évêque de Québec, tous ceux qui furent élevés à cette dignité pendant cet intervalle. - 3o Quoique depuis un petit nombre d'années le Gouvernement, forcé par les circonstances, ait reconnu un évêque de Québec, il n'a pas renoncé pour cela à sa prétendue Suprématie qui lui attribue la nomination du même. 4o Il employa en deux ou trois circonstances, sans succès, il est vrai, des moyens presque violents pour placer sur ce Siège des sujets de son choix et bien peu dignes des hautes fonctions de l'Episcopat. ${ }^{2} 8$ 5o Il nomma dans une autre occasion un individu qui n'avait jamais vu le Canada et qui probablement était étranger à la langue du pays. 60 ...Ses représentants ne pouvant avec prudence contrarier le vœu du clergé et du peuple, et entraver la marche des lois du pays, s'accordèrent en secret avec les Evêques..., tandis que publiquement ils soutenaient avec les employés du Gouvernement qu'il ne pouvait y avoir en Canada d'Evêque Catholique qu'en vertu de la suprématie du Roi... - 7o ...Le gouvernement si tard qu'en 1825 et 1832 a eu recours à une nouvelle manœuvre pour arriver à son but. A ces deux époques les gouverneurs généraux des Canadas, qui jusque là avaient toujours approuvé au nom du roi les coadjuteurs élus, les référèrent au bon plaisir de Sa Majesté même... .

28. M. Maguire fait allusion ici d'abord a l'ingérence de Lord Sydney qui, en 1784, voulut faire nommer à la place de $\mathbf{M g r}$ Hubert, $M$. de Montgolfier, alors Agé de 73 ans; puis à celle du Duc de Kent qui s'opposa fermement a l'élection de Mgr Plessis et proposa de nommer à sa place un vieux curé incapable d'exercer les fonictions de l'épiscopat. (Voir: Mgr Henri Têtu, Les Eveques de Quebec, (Granger et Mame, 1930, éd. populaire), t. III: 14, 66.) 
A la fin de son exposé, M. Maguire fait remarquer très justement que les prétentions du gouvernement anglais " sont excusables et jusqu'à un certain degré méritent quelqu'indulgence ", parce que les lois civiles et ecclésiastiques supposent un évêque et un chef d'état qui collaborent étroitement à leur exécution. Qu'adviendrait-il de la religion, si le gouvernement " refusait de reconnaître les évêques au choix desquels il n'aurait concouru en aucune manière?"

Toute cette démonstration n'avait qu'un but: montrer la nécessité des "tempéramens conciliatoires. " Après tout une seule chose importait: que l'archevêque pat, à son avènement, présenter au gouverneur un candidat à l'épiscopat. Pour cela, il suffirait que le SaintSiège approuvât d'avance les trois sujets recommandés par l'archevêque. En conséquence, M. Maguire suppliait le Souverain Pontife de régler les élections des coadjuteurs de la façon suivante:

- lo que trois personnes d'un mérite distingué et dignes de l'épiscopat soient recommandées toujours d'avance par l'Archevêque au S. Siège pour en recevoir l'approbation. 2o Qu'advenant le décès de l'Archevêque son successeur présente à l'agrément de Sa Majesté Britannique l'une des trois personnes ainsi approuvées du S. Siège, la proclame au diocèse et postule en Cour de Rome les provisions d'usage. - 3o Que l'époque de la réception de ces provisions soit toujours celle où l'evêque se mettra en devoir de recommander au S.-Siège trois personnes pour l'élection suivante. 4o Que dans la circonstance où l'Archevêque ou son Coadjuteur décèderaient avant qu'on connût au Canada l'approbation des trois dernières personnes recommandées, l'Archevếque put alors offrir à l'agrément du Représentant du roi, l'une des deux personnes déjà agr@ês du S.-Siège pour la dernière Glection ${ }^{29}$.

Malgré sa solidité et son intérêt, cette pétition produisit peu d'effet, parce qu'elle laissait de côté le point délicat sur lequel s'opposaient l'archevêque de Québec et le Séminaire de Montréal, ${ }^{3}{ }^{\circ}$ savoir:

29. Pétition de M. Maguire a Grégoire XVI, avril 1834 AAM.

30. Saint-Sulpice est-il intervenu dans cette affaire pour des motifs d'intérêt puremen't local? Il est permis d'en douter a la suite de cette information recueillie par M. Maguire durant son séjour à Rome: "...Saint-Sulpice de Paris... depuis quelques années, nomme à tous les évêchés de l'Amérique du Nord. J'avais déjâ, avant mon départ du Canada, soupçonné cette influence de Saint-Sulpice de Paris. Aujourd'hui, le Dr England, évêque de Charlestown, me l'a prouvée jusqu'à l'évi- 
le mode de désignation au Canada même des trois candidats à l'épiscopat. Cette lacune explique en grande partie la décision des Cardinaux qui, dans la congrégation du 21 juillet, rejetèrent les propositions de $M$. Maguire et recommandèrent fortement le projet de $M$. Thavenet.

Heureusement, un événement imprévu permit à l'agent de l'évêque de Québec de compléter à temps son mémoire. Il était de règle de garder le secret sur les communications faites à la Propagande par les deux parties. Or le 24 juillet, comme entraîné par " un instinct irrésistible ", M. Maguire se rendit chez Mgr Mai qui de prime abord lui dit: « J'ai appris qu'un cardinal indiscret a communiqué vos pétitions et mémoires à l'abbé Thavenet; or, il est juste, pour balancer les comptes, que vous ayez à votre tour communication du mémoire de $M$. Thavenet; le voilà; lisez "). ${ }^{31}$ C'est ainsi que M. Maguire put prendre connaissance du plan de l'agent de Saint-Sulpice. Ce dernier proposait que " pour les élections futures des coadjuteurs de Québec, il soit réglé: que les Suffragants, les Grands-Vicaires et les Supérieurs des Séminaires de Québec et de Montréal transmettent chacun trois noms à l'êvêque de Québec; que celui-ci ajoute tel nombre qu'il voudra, et qu'il fasse parvenir au S.-Siège une liste de tous ces noms, afin que le S.-Siège fasse le choix qu'il lui plaira 1$).^{32}$

M. Maguire vit tout de suite le danger que renfermait ce projet et, séance tenante, il le combattit en démontrant que Saint-Sulpice saurait toujours commuaiquer au Saint-Siège les noms des personnes nommées par son supérieur, et qu'il vaudrait aussi bien, dans ce cas, abandonner tout de suite au seul supérieur de Montréal la nomination du coadjuteur de Québec. Il quitta Mgr Mai sans l'avoir convaincu. De retour chez lui, " pensif et inquiet 》, il se décida à mettre par écrit ses arguments dans l'espoir qu'ils produiraient une plus forte impression. Dans cette nouvelle lettre, après avoir rappelé brièvement les

dence". (Lettre à Mgr Sigriay, 15 juin 1834). On aurait là l'explication, au moins pour les Etats-Unis, de la nomination, à cette époque, d'évêques français, sulpiciens pour la plupart, tels que: Mgr Flaget a Bardstown en 1807, Mgr de Cheverus a Boston en 1810 (devenu plus tard cardinal et archevêque de Bordeaux), Mgr Dubourg à la Nouvelle-Orléans en 1815, Mgr Maréchal à Baltimore en 1817, Mgr David à Bardstown en 1818, Mgr Dubois à New-York en 1826, Mgr Bruté de Rémur à Vincennes, en 1834, Mgr Chabrat à Bardstown en 1834.

31. Maguire a Signay et a Lartigue, 23 juillet 1834, AAM.

32. Ibidem. 
conclusions générales de sa pétition précédente: la nécessité d'éviter toute collision avec le gouvernement britannique et d'avoir toujours prêt, à la mort de chaque archevêque, un nouveau coadjuteur à présenter, il demandait de laisser à l'archevêque et à son conseil le choix sur les trois personnes approuvées par Rome. Quant au conseil, il serait composé des évêques suffragants et des grands vicaires du diocèse, en tout onze personnes. Les séminaires de Québec et de Montréal y seraient suffisamment représentés puisqu'ils avaient chacun leur grand vicaire. ${ }^{3} 3$

Le 25 juillet, M. Maguire porta sa lettre à $\mathrm{Mgr}$ Mai qui parut très satisfait de ses nouvelles suggestions et crut même devoir les mettre sous les yeux du Saint-Père. A l'audience du 27 juillet, le secrétaire de la Propagande, malgré la recommandation contraire des Cardinaux, se fit auprès du Souverain Pontife l'ardent défenseur du projet de M. Maguire. Après deux heures de discussion, Grégoire XVI s'arrêta au plan suivant: "Les Evêques Suffragants et les Grands Vicaires (et les supérieurs des Séminaires de Québec et de Montréal, quand il n'y aura pas de Grands Vicaires dans les Maisons) s'assembleront et voteront par scrutin. - Il sera libre à ceux d'entre eux qui ne pourront pas se rendre à l'assemblée d'y faire parvenir leurs notes par lettres missives. - L'Archevêque recommandera au S.-Siège les trois personnes qui auront réuni le plus de suffrages. - L'Archevêque aura le choix du coadjuteur sur les trois personnes approuvées de Rome, et le pourra présenter dans le temps convenable au représentant de Sa Majesté Britannique ". 34

Sur ce point encore, M. Maguire ne pouvait espérer une solution plus favorable.

Une dernière question restait à résoudre, l'érection du Diocèse de Montréal ou la démission de Mgr Lartigue, évêque de Telmesse, auxiliaire et suffragant de l'évêque de Québec pour le district de Montréal.

On connaît les difficultés que ce prélat eut à surmonter dès le début de son épiscopàt, une partie de son clergé et de ses ouailles,

33. Maguire à Mgr Mai, 25 juillet 1834, AAM.

34. Maguire à Signay et à Lartigue, 28 juillet 1834, AAM. 
le Séminaire de Saint-Sulpice en tête, refusant, non sans quelque raison, de lui reconnaitre les droits et les honneurs dus à un évêque suffragant. Pour dissiper cette opposition, il ne voyait qu'un moyen: la nominatỉbn d'un évêque titulaire à Montréal. Durant dix ans, il travailla vainement à la réalisation de son projet; à Québec, à Londres, à Rome surgissaient sans cesse des oppositions apparemment insurmontables.

Se croyant alors lui-même un obstacle au rétablissement de la paix, il songea sérieusement à donner sa démission. Profitant du voyage de MM. Tabeau et Maguire, à Rome, en 1829-1830, il les chargea de plaider sa cause auprès du Souverain Pontife. Il les prévint que s'ils ne pouvaient pas obtenir l'établissement d'un diocèse à Montréal, ils devraient solliciter l'acceptation de sa démission de toutes leurs forces. Le Saint-Siège ne jugea pas à propos de se rendre à leur demande, ce qui n'empêcha pas Mgr Lartigue de la renouveler chaque fois qu'il eut l'occasion d'écrire dans la suite à la Propagande.

En 1834, la révélation des intrigues de $M$. Thavenet en Cour de Rome l'amena à modifier sensiblement sa requête. Dans la crainte de la suppression du poste de suffragant pour le district de Montréal, il retira sa démission donnée jusque-là sans condition, mais pour la présenter aussitôt sous une autre forme. Par l'intermédiaire de $\mathbf{M}$. Maguire, il la remettait " entre les mains du Souverain Pontife ou du Collège des Cardinaux a la condition sine qua non que Montréal soit érigé en siège épiscopal ou qu'un autre suffragant jouissant des mêmes privilèges lui succède à Montréal; et que ce nouveau suffragant soit celui que l'Archevêque de Québec a déjà proposé au Souverain Pontife $1 .^{35}$ Ce nouveau candidat était un vicaire général du district de Montréal, M. Antoine Tabeau, que M. Thavenet avait déjà présenté a la Propagande comme un ennemi de Saint-Sulpice. Il n'en fallait pas davantage pour que les Cardinaux, par ailleurs très mécontents de la condition sine qua non de la démission, rejettent la demande de Mgr Lartigue, décision aussitôt sanctionnée par le Saint-Père.

Toutefois, un refus aussi catégorique ne devait pas décourager l'agent de l'évêque de Québec. Il revint à la charge auprès de Mgr Mai, qui, toujours sympathique à la cause des évêques, l'invita à mettre

35. Lartigue à Maguire, 25 et 29 juillet 1834, Rapport de l'Archiviste de la Province de Québec, (1943-1944): 262-263. 
par écrit ses observations en lui promettant de les faire valoir de son mieux dans la prochaine audience. M. Maguire écrivit alors un dernier mémóire dans lequel il suppliait le Souverain Póntife de "venir au secours de l'Evêque de Télmesse pour le délivrer de la po'sition violente et contre nature où il se trouvait engagé depuis tant d'années" . Laissant de côté presque complètement la question de la démission, il insistait surtout sur la nécessité d'établir un diocèse à Montréal. Pour donner plus de force à sa démonstration il ne trouvait rien de mieux que de citer de larges extraits de la dernière lettre de $\mathbf{M g r}$ Lartigue dont voici le passage le plus important:

Pour ce qui est d'un évêché à Montréal, il est aisé de voir que jamais les troubles ne finiront dans ce district sans qu'il y ait un évêque indépendant de celui de Québec, qui à cette distance (180 milles) ne peut voir l'état des choses ni se faire obøir; l'expérience de 13 ans en a fourni assez de preuves... Si l'on a pu donner dix raisons pour l'erection de ces deux évêchés (Charlottetown et Kingston) il y en a cent a alleguer pour celui de Montréal: la population de mon district qui dépasse de beaucoup celle de tout le reste du BasCanada ensemble; l'étendue de ce même district qui a plus de 150 lieues (450 milles) en longueur; mes forces actuelles qui ne me permettent plus de visiter qu'en six ans tout mon district, tandis que je le pourrais faire en trois ans avec un coadjuteur... une foule d'autres motifs montrent l'urgence de cet établissement.

M. Maguire attirait ensuite l'attention sur le noble désintéressement de l'évêque de Telmesse qui se disait prêt à céder la place à un autre, si la répugnance qu'on aurait en Angleterre à ériger Montréal en évêché « venait de raisons politiqúes qui lui fussent personnelles 》. ${ }^{36}$ Enfin il notait que l'arahevêque de Québec appuyait entièrement son auxiliaire même pour la condition sine qua non de sa démission.

Quand, le 31 juillet, M. Maguire se présenta chez Mgr Mai pour lui porter sa lettre, il le trouva fort embarrassé. Le Secrétaire de la Propagande jugeait máaintenant très difficile pour lui de reprendre la discussion d'une affaire si définitivement réglée par le Souverain Pontife. Il l'invita à plaider lui-même sa cause, lui promettant de

36. Mgr Lartigue fait ici allusion à son lien de parenté avec Louis-Joseph Papineau, chef du parti patriote. 
l'aider, s'il réussissait, en faisant valoir les nouveaux arguments contenus dans sa lettre. Trois semaines plus tard, M. Maguire était reçu en audience privée par Grégoire XVI qui lui faisait un accueil très paternel et consentait volontiers à reprendre la cause de l'Evêque de Telmesse. La question fut enfin réglée au cours de septembre dans un sens à peu près conforme à la requête de M. Maguire. M. Antoine Tabeau était nommé évêque, ${ }^{37}$ mais comme " vice-gérant avec droit de succession " et non comme remplaçant de Mgr Lartigue; celui-ci gardait sa fonction aussi longtemps qu'il serait capable de l'exercer avec l'aide d'un auxiliaire. ${ }^{38}$ Le Souverain Pontife avait pris cette décision, parce qu'il avait l'espoir d'établir avant longtemps un diocèse à Montréal.

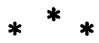

Après ce dernier succès, le délégué de l'évêque de Québec avait bien le droit de présenter avec fierté le bilan de son voyage: "Votre Grandeur, écrivait-il à Mgr Signay, aura déjà su par mes dernières lettres que les objets de ma mission sont accomplis et au-deld: 10 - un coadjuteur obtenu pour Québec. 2o - La démission de Mgr de Telmesse et un suffragant pour le remplacer. ${ }^{30} 30-$ Un mode pour les élections futures des coadjuteurs de Québec... 4o - Un ordre à SaintSulpice de n'aliéner ses biens que du consentement de l'archevêque.... 40 M. Maguire ne mentionnait là que les résultats immédiats de sa mission. Quelle satisfaction n'aurait-il pas éprouvée s'il avait pu en prévoir les répercussions lointaines. Pour l'Eglise du Canada ce fut une plus large autonomie. Grâce au nouveau mode d'élection des coadjuteurs, il ne fut plus question de se défendre contre l'ingérence indue du pouvoir civil ou d'autres institutions dans la nomination des évêques. D'autre part, la décision de Rome relative aux biens de SaintSulpice mit fin aux tentatives du gouvernement anglais pour la pos-

37. M. Tabeau tomba gravement malade en janvier 1835 et mourut en mai de la même année, avant d'avoir reçu la consécration épiscopale.

38. Mgr Lartigué à Mgr Flaget, évêque de Bardstown, 7 avril 1835, Rapport de l'Archiviste de la Province de Québec, (1943-1944): 292-293.

39. Avant d'écrire sa lettre, M. Maguire n'avait évidemment pas lu les bulles de M. Tabeau le nommant "vice-gérant" de Mgr de Telmesse.

40. Maguire à Signay et Tabeau, 8 nov. 1834, AAM. 
session des biens ecclésiastiques. Enfin, pour Montréal, la conséquence la plus importante fut l'érection du district en diòcèse. ${ }^{41}$

Avant de quitter Rome, M. Maguire écrivit une dernière lettre au Souverain Pontife " pour lui offrir respectueusement l'expression de la gratitude éternelle du diocèse de $Q u e ́ b e c$ pour les grâces insignes qu'il a daigné lui accorder $).{ }^{42}$ Cette démarche n'en est pas une de simple politesse. En définitive, c'est bien grâce à la sagesse et à la générosité de Grégoire XVI si, en cette occasion, l'Église du Canada fit un pas décisif dans la voie du progrès et de la liberté. Mais ceci ne doit pas faire oublier le principal artisan de ce magnifique résultat. Rendons hommage au prêtre méconnu ${ }^{43}$ qui, en des circonstances parfois très pénibles, mit au service d'une cause juste et sacrée tout son talent et toute son énergie.

\author{
Antonio Dansereau, p.s.s. \\ Collège de Montréal, \\ membre de la Société d'histoire \\ du Diocèse de Montréal
}

\begin{abstract}
41. Il en coata bien des démarches à $M$. Maguire avant de connaitre la marche à suivre pour arriver à ce résultat. Il apprit enfin de source officielle qưe "Rome ne s'occuperait nullement de cette affaire aussi longtemps que l'évêque de Québec n'en ferait pas la demande" (lettre à Mgr Lartigue, 6 juillet 1834). Mgr Lartigute, aussitôt informé, pressa Mgr Signay de faire les démarches nécessaires sans réussir à obtenir autre chose que des promesses. Il fallut, à la fin de l'année 1835, une requête de tout le clergé du district pour décider l'évêque de Québec à agir. Mais, par crainte du gouvernement, il crut nécessaire d'envoyer la requête du clergé au gouverneur avant de l'adresser à Rome. Ce détour aurait retardé l'affaire indéfiniment, si Mgr Lartigue, qui avait hâte d'en finir, n'eât trouvé un moyen de sortir de l'impasse. Il chargea Mgr Provencher, en route pour Rome, de présenter à la Propagande, avec une lettre d'assentiment de Mgr Signay reçue au cours de l'été, une copie de la requête du clergé. Quelques jours après la réception de ces documents, le Souverain Pontife signait le décret d'érection du diocèse de Montréal et Mgr Lartigue en devenait le premier titulaire. (Voir la correspondance de Mgr Lartigue dans le Rapport de l'Archiviste de la province de Quebec, (1943-1944), passim).
\end{abstract}

42. Maguire à Sa Sainteté Grégoire XVI, 2 déc. 1834, AAM.

43. Il n'est fait mention du voyage de 1833-1834 ni dans le Dictionnaire général du Canada du Père Lejeune, ni dans le Dictionnaire biographique du clergé canadien-francais du chan. J.-B. Allaire. 